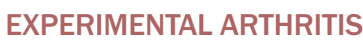

\section{CPB2 dampens inflammation in autoimmune arthritis}

The fibrinolysis inhibitor carboxypeptidase B2 (CPB2, also known as plasma carboxypeptidase $\mathrm{B}$ ) downregulates C5a-mediated inflammation in experimental models of autoimmune arthritis, and patients with rheumatoid arthritis (RA) who possess a CPB2 variant with a longer half-life are at a reduced risk of developing severe RA. These new findings, published in The Journal of Clinical Investigation, demonstrate the protective effects of CPB2 in autoimmune arthritis.

"CPB2 can have anti-inflammatory or procoagulant activity," notes senior author William Robinson, Stanford University, USA. As the proinflammatory mediators C5a, osteopontin and bradykin are involved in RA pathogenesis and have been shown to be substrates of CPB2 in vitro, Robinson and colleagues wanted to determine the role of CPB2 in RA in vivo.

After induction of arthritis with anticollagen antibodies, the researchers found that inflammatory arthritis in mice lacking CPB2 was exacerbated compared with that in wild-type mice. Moreover, injection of the cleaved form of C5a (C5a-desArg; by treating with CPB2) into the peritoneum and joints of wild-type mice induced less inflammation and immune cell recruitment than injection with intact C5a.

Interestingly, patients with RA who have a CPB2 variant with a long half-life (1040T
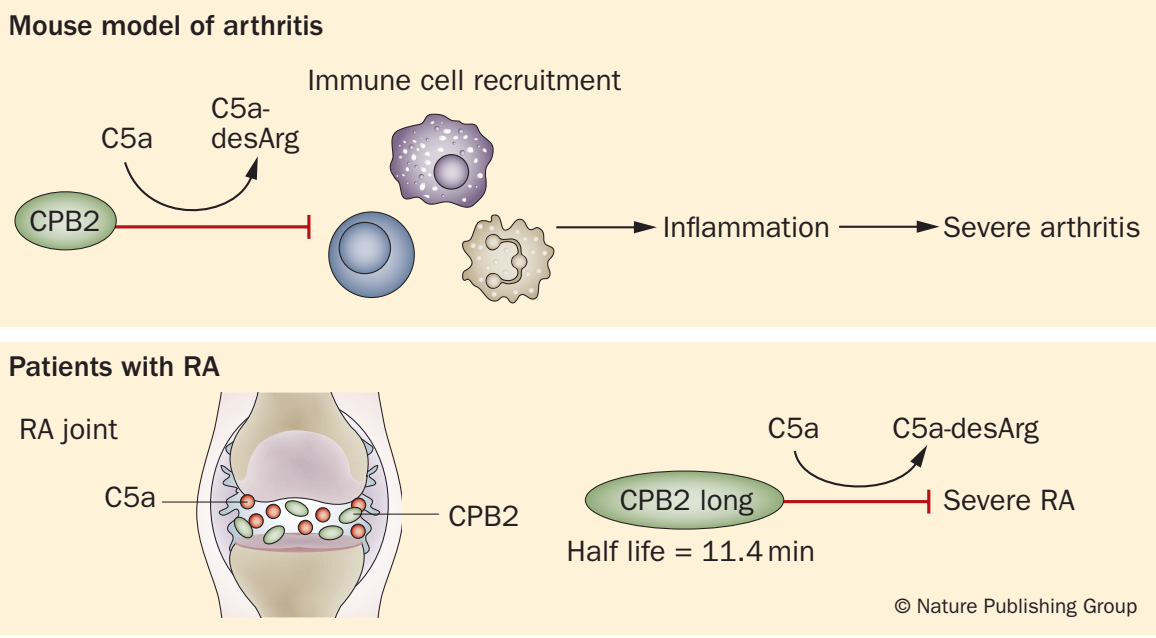

allele) were less likely to develop severe RA than those who have the $1040 \mathrm{C}$ allele (shorter half-life CPB2); in vitro, the long half-life CPB2 neutralized C5a activity more efficiently than the shorter half-life counterpart. Furthermore, both CPB2 and C5a levels were elevated in the synovial fluid of patients with RA, and CPB2 was locally produced in RA joints by synoviocytes and immune cells.

Robinson and colleagues now plan to investigate the role of CPB2 in other inflammatory conditions and in the erosion of bone and cartilage. Furthermore, CPB2 could be used as a genetic biomarker for RA, claim the authors. " $C P B 2$ genotyping could potentially be used to screen individuals who are at risk of progressing to a severe, erosive form of the disease, or to predict the response of patients with RA to therapeutic inhibitors targeting CPB2 substrates, such as anti-C5a therapy," adds Robinson.

Katrina Ray

Original article Song, J. J. et al. Plasma carboxypeptidase $B$ downregulates inflammatory responses in autoimmune arthritis. J. Clin. Invest. doi:10.1172/JCI46387 